\title{
Direct Observation of Bloch Harmonics and Negative Phase Velocity in Photonic Crystal Waveguides
}

\author{
H. Gersen, ${ }^{1, *}$ T. J. Karle, ${ }^{2}$ R. J. P. Engelen, ${ }^{1}$ W. Bogaerts, ${ }^{3}$ J. P. Korterik, ${ }^{1}$ N. F. van Hulst, ${ }^{1}$ T. F. Krauss, ${ }^{2}$ and L. Kuipers ${ }^{1,4, \dagger}$ \\ ${ }^{1}$ Applied Optics Group, Department of Science and Technology and MESA ${ }^{+}$Institute for Nanotechnology, University of Twente, \\ P.O. Box 217, 7500 AE Enschede, The Netherlands \\ ${ }^{2}$ School of Physics and Astronomy, University of St. Andrews, St. Andrews, Fife KY16 9SS, Scotland \\ ${ }^{3}$ Ghent University-IMEC, Department of Information Technology (INTEC), B-9000 Gent, Belgium \\ ${ }^{4}$ FOM-Institute for Atomic and Molecular Physics (AMOLF), Kruislaan 407, 1098 SJ Amsterdam, The Netherlands
}

(Received 27 June 2004; published 30 March 2005)

\begin{abstract}
The eigenfield distribution and the band structure of a photonic crystal waveguide have been measured with a phase-sensitive near-field scanning optical microscope. Bloch modes, which consist of more than one spatial frequency, are visualized in the waveguide. In the band structure, multiple Brillouin zones due to zone folding are observed, in which positive and negative dispersion is seen. The negative slopes are shown to correspond to a negative phase velocity but a positive group velocity. The lateral mode profile for modes separated by one reciprocal lattice vector is found to be different.
\end{abstract}

DOI: 10.1103/PhysRevLett.94.123901

The ability to control the flow of light in photonic crystals (PhCs) is both scientifically and technologically important. Optical waves propagating in a $\mathrm{PhC}$ experience a spatially periodic modulation of the index of refraction and, as a result, exhibit behavior that is similar to electrons in a semiconductor crystal [1]. PhCs have the capacity of drastically affecting the optical dispersion relations, symmetries, and spatial distribution of electromagnetic modes and thereby provide a new dimension in controlling the flow of light [2].

Incorporation of structural defects into $\mathrm{PhCs}$ allows the implementation of various functional components [2]. Arguably the most important type of defect guides in $\mathrm{PhCs}$ is a straight guide with one or more rows of holes missing [3-5]. Properly designed, these missing rows form a $\mathrm{PhC}$ waveguide $(\mathrm{PhCW})$ which allows efficient guiding of light within a photonic band gap. Because of the periodicity imposed on such a waveguide, its guided modes obey the Bloch theorem [6,7]. This results in an intricate interplay between the guided modes and their local environment. A major practical obstacle in experimentally unravelling this interplay is the low signal levels involved combined with Fabry-Perot oscillations often resulting from poorly matched interfaces on the sample [8]. To understand the intricacies of light propagation in such a system and test theory, imaging the flow of light is highly advantageous.

Conventional imaging of a PhCW has been found suitable for mapping the dispersion of leaky $\mathrm{PhCW}$ modes exploiting the interference between counterpropagating waves [9]. However, direct information concerning the field distribution of the modes being present is not obtained, as the resolution of this method is intrinsically limited by diffraction. Furthermore, true guided $\mathrm{PhCW}$ modes have a propagation constant larger than that in air for the same optical frequency. Therefore they cannot be
PACS numbers: 42.70.Qs, 42.25.-p, 68.37.Uv, 78.67.-n

observed with far-field techniques because their field in air is evanescent, i.e., exponentially decaying over distances of the order of the light wavelength. Evanescent fields can be detected only by a local probe immersed into the near field of the sample with a collection mode near-field optical microscope (NSOM) [10].

It has been demonstrated numerically that highresolution NSOM images of intensity distributions can portray the path that light takes in the crystal. As a result of counterpropagating Bloch waves in finite structures, also wave vector dispersion information can be obtained [11]. To date, several exciting NSOM experiments have been performed on $\mathrm{PhCs}$, none of which directly probe the eigenmodes of the $\mathrm{PhC}$ waveguide or the wave vector dispersion diagram [12-15].

In this Letter the local measurement of the band structure in a $\mathrm{PhCW}$ is demonstrated with a phase-sensitive heterodyne NSOM. Bloch modes consisting of more than one spatial frequency are measured, revealing multiple Brillouin zones due to zone folding. In addition, the underlying lateral mode structure of all the $\mathrm{PhCW}$ modes present is determined. We find that the modal distribution for lines separated by the reciprocal lattice vector is not identical.

The majority of NSOM experiments detect local intensity distributions. However, by interfering the light collected by the optical probe with a frequency shifted reference beam from the same laser source, one can obtain the local field amplitude and phase (see Fig. 1) [16-18]. By detecting the interference signal with a lock-in amplifier (LIA), the $X$ and $Y$ quadratures of the LIA signal provide the cosine and sine of the phase difference between the NSOM light and the reference beam [17]. The experimental arrangement used consists of a heterodyne timeresolved collection mode NSOM as described elsewhere $[19,20]$. To prevent coupling of spurious stray light, the optical fiber tip is metal coated with an aperture of $240 \mathrm{~nm}$ 
(a)

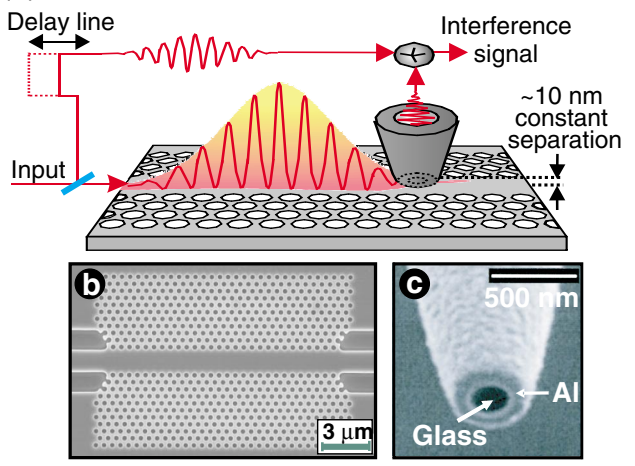

FIG. 1 (color). (a) Schematic representation of a phasesensitive NSOM experiment on a W3 PhCW. The evanescent field of a pulse traveling inside the photonic structure is picked up by a coated fiber probe with a subwavelength aperture as shown in (c). At each position the signal picked up by the probe is interferometrically mixed with part of the same pulse that has propagated through the reference branch giving access to the local field amplitude and phase. (b) A scanning electron micrograph of the sample under study.

at the end, as shown in Fig. 1(c). The integrity of the probes is verified after the measurements by scanning electron microscopy. By using short laser pulses the background resulting from Fabry-Perot reflections and scattering out of the structure can be completely suppressed by discrimination in the time domain.

The structure under investigation is fabricated in a silicon-on-insulator wafer, comprising a $220 \mathrm{~nm} \mathrm{Si} \mathrm{core}$ on top of a $1 \mu \mathrm{m} \mathrm{SiO}{ }_{2}$ cladding [21]. Holes are arranged in a hexagonal array (period $a=460 \mathrm{~nm}$, hole radius $r=$ $130 \mathrm{~nm}$ ), and three rows of missing holes define the socalled W3 PhCW [see Fig. 1(b)]. The PhCW displayed in Fig. 1(b) is shorter than the one used in the experiments (32 versus 256 crystal periods), but allows all the relevant features of the structure to be seen in detail. Linearly TEpolarized light pulses (E $\|$ to crystal plane, pulse duration at FWHM $123 \mathrm{fs}$ ) are launched into the $\mathrm{PhCW}$. Our results have been reproduced on multiple samples using different probes.

The result of a local phase-sensitive heterodyne interference measurement on a W3 PhCW is shown in Fig. 2. Figure 2(a) shows the measured topography. The access and exit waveguides are visible at the left and right sides of the image, and the hole pattern that forms the $\mathrm{PhC}$ can be distinguished. Figure 2(b) shows the raw data of one of the LIA signals, which corresponds to the local optical amplitude times the cosine of the phase [20]. The color scale runs from black to bright yellow for large negative and positive signal values, respectively. Such an image contains all the information of the spatial frequencies of the modes excited in the waveguide. In Fig. 2(b) a large spread in spatial frequencies is clearly visible. From the two LIA signals the optical amplitude can be separated [depicted in

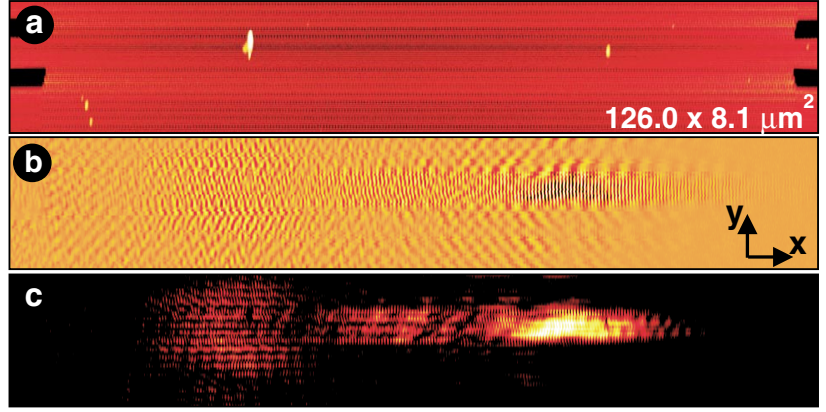

FIG. 2 (color). A phase-sensitive NSOM measurement on a W3 PhCW for a fixed position of the optical delay. Short pulses were launched at a wavelength of $1245 \pm 0.5 \mathrm{~nm}(\omega=0.369$; $a=460 \mathrm{~nm}$ ). (a) Topography of the structure. (b) Raw data from the lock-in amplifier corresponding to the amplitude times the cosine of the optical field. In the waveguide, guided light with a large spread in spatial frequencies is visible. (c) The optical field amplitude. It is apparent that pulses with different modal distributions are excited in the W3 waveguide. The dominant periodicity observed in (c) corresponds to $460 \mathrm{~nm}$, as expected for Bloch modes.

Fig. 2(c)]. It is apparent that pulses with different modal distributions are excited in the PhCW. These pulses are separated after only a short propagation length, a clear indication of the strong dispersive properties of the $\mathrm{PhCW}$. The dominant periodicity observed in Fig. 2(c) corresponds to $460 \mathrm{~nm}$, as expected for Bloch modes in periodic media with that period [22].

A spatial Fourier transform in the $x$ direction of the data displayed in Fig. 2(b) for one particular $y$ yields the spectrum $F\left(k_{x}\right)$ of the spatial frequencies $\left(k_{x}\right)$ [17]. The measurements and subsequent Fourier analysis are repeated for different wavelengths ranging from 1180 to $1345 \mathrm{~nm}$ in steps of $5 \mathrm{~nm}$. To improve signal-to-noise ratio, the spectra $F\left(k_{x}\right)$ are summed for all $y$. Figure 3(a) shows the band structure constructed from the measured spatial frequencies. The vertical axis displays the excitation wavelength in terms of $\omega$, while the horizontal axis corresponds to the observed spatial frequencies. The finite resolution $\omega$ is determined by the finite coherence length of the pulses used. Our local measurement technique reveals zone folding as multiple Brillouin zones are observed.

In addition to probing the band structure of the $\mathrm{PhCW}$ modes, the use of a phase-sensitive NSOM allows the determination of the underlying mode structure of all the $\mathrm{PhCW}$ modes present. The lateral field profile of a mode is obtained by determining the amplitude of the peak in the $F\left(k_{x}\right)$, corresponding to that mode, as function of $y[16,17]$. By displaying $F\left(k_{x}\right)$ for all values of $y$, the field profile can be displayed for all modes at the same time. The result of this Fourier analysis for $\omega=0.369$ as presented in Fig. 2(b) is displayed in Fig. 3(b). The $F\left(k_{x}\right)$ spectra between $k_{x}=1$ and 2 are multiplied by 5 to enhance visibility of weak modes in the 2 nd Brillouin zone. A 


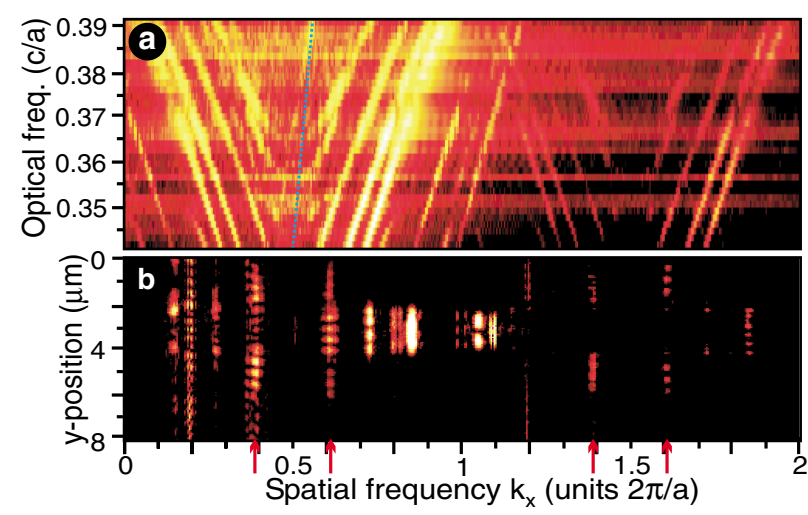

FIG. 3 (color). (a) Experimentally mapped dispersion diagram of the modes excited in the W3 waveguide. Displayed is the Fourier transform of the data for multiple excitation wavelengths on a logarithmic scale. Clearly modes below and above the light cone (dotted line) are visible. (b) Displays the lateral mode distribution for $\omega=0.369$ by displaying $F\left(k_{x}\right)$ for each $y$ in Fig. 2(b) on a linear scale. Here we can directly see the modal distribution for each of the lines in the dispersion diagram at $\omega=0.369$. It is clear that for Bloch harmonics that belong to the same Bloch mode, e.g., indicated by arrows, the modal distribution is not the same.

vertical line for a certain spatial frequency thus corresponds to the lateral mode profile for the mode corresponding to that $k_{x}$. As a result, Fig. 3(b) directly displays the modal distribution of all PhCW modes for $\omega=0.369$. Both modal distributions that are completely confined to the waveguide regions (see, e.g., near $k_{x}=0.8$ ) and with significant field tails in the $\mathrm{PhC}$ are observed (see, e.g., $\left.k_{x}=0.6\right)$ [23].

Bloch modes contain more than one spatial Fourier component. These Bloch harmonics are separated by $2 \pi / a$, where $a$ is the periodicity. The separation between the different plane waves components is what generates the dominant periodicity of $460 \mathrm{~nm}$ visible in Fig. 2(c). The $2 \pi / a$ separation can be seen in Fig. 3(a) where we displayed the first two Brillouin zones. Note that a Fourier transform is not sensitive to the sign of $k$ and therefore folds $-k$ onto the positive axis. As a result, an example of Bloch harmonics that belong to the same Bloch mode for $\omega=0.369$ can be given as indicated by the arrows in Figs. 3(b) and 4(a). Figure 4(a) depicts the entire Fourier spectrum $F\left(k_{x}\right)$ for $\omega=0.369$ on a logarithmic scale. In the literature, lines separated by an integer number of reciprocal lattice vectors are often considered to be identical. However, if we look at Fig. 3(b) at the mode patterns of, for example, the wave vectors 0.607 and 1.603 , we see that they are not the same. The differences in mode patterns for individual wave vectors become clearer when the mode profiles are displayed in a graph [Fig. 4(b)]. For example, comparing the mode profiles for $k_{x}=0.607$ and $k_{x}=$ 1.603 shows that the profile for $k_{x}=1.603$ has no field amplitude at the location of the PhCW $(2.5<y<4 \mu \mathrm{m})$,
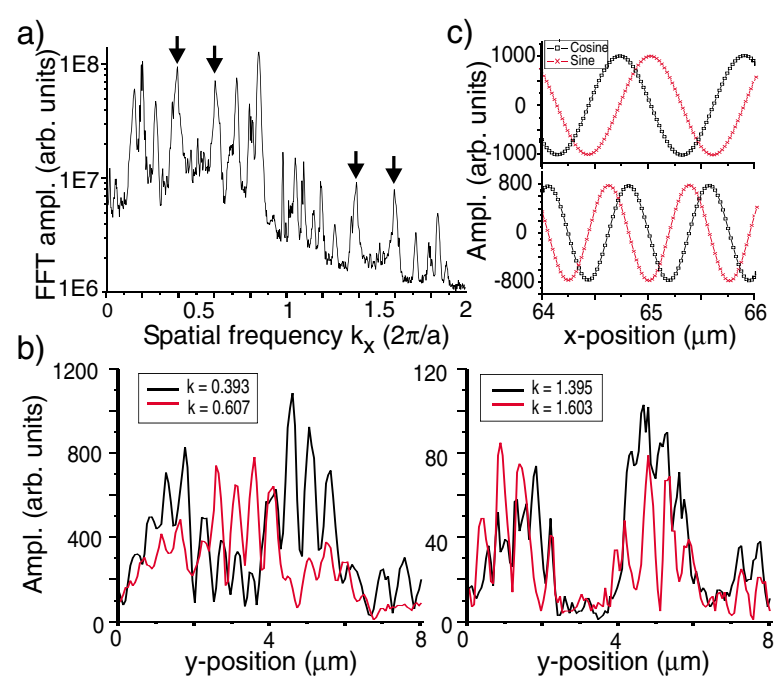

FIG. 4 (color). (a) Spatial Fourier spectrum $F\left(k_{x}\right)$ for $\omega=$ 0.369 summed over all $y$. Arrows indicate peaks that belong to the same Bloch mode (FFT $=$ fast Fourier transform). (b) Lateral mode profiles for the Bloch harmonics indicated by the arrows in Fig. 3(b) and in panel (a). The modal distribution is different for each individual Bloch harmonic. (c) A Fourier filter allows retrieval of the phase evolution of individual modes. Top panel in (c) shows the sine and cosine channel of the LIA for $k_{x}=0.393$, while the bottom panel corresponds to $k_{x}=0.607$. For the Bloch harmonic at $k_{x}=0.393$ the sine is in front, while it is lagging at $k_{x}=0.607$. As a result, the wave vectors for these two harmonics from the same Bloch mode have an opposite sign.

whereas $k_{x}=0.607$ does. In Fig. 3(b) this effect is also visible for the Bloch harmonics of other Bloch modes. The experimental data therefore show that the modal distributions for Bloch harmonics separated by $2 \pi / a$ are different. The exact physical interpretation behind this observation is not yet clear. We suggest that (part of) the explanation should be sought in the fact that the spatial overtones (higher Bloch harmonics) are generated at positions where the light probes the periodic potential arising from the crystal. Hence, the higher harmonics are stronger in the crystal region [Fig. 4(b), right panel], whereas the fundamental also has amplitude in the area without holes [Fig. 4(b), left panel, $2.5<y<4 \mu \mathrm{m}$ ].

In one Brillouin zone in Fig. 3(a), lines with both negative and positive slope are visible as a result of zone folding. For the Bloch harmonics at $k_{x}=0.393$ and $k_{x}=$ 0.607 , the cosine and sine signal from the LIA are shown in the top and the bottom panel of Fig. 4(c), respectively. These data are retrieved by an inverse Fourier transform after applying a Gaussian filter centered on the desired peak. It is clear that in the top panel $\left(k_{x}=0.393\right)$ the sine is in front, while in the bottom panel $\left(k_{x}=0.607\right)$ the sine is lagging. As the LIA signal provides the cosine and sine of the phase difference between the light in the $\mathrm{PhCW}$ and the reference light, conventional goniometry [24] shows that the wave vectors for the two Bloch har- 
monics have an opposite sign. It is interesting to observe that the energy of the Bloch mode propagates in a direction opposite to the $k$ vector for one of these modes [25]. Because this mode is referenced by this $k$ vector, the group velocity is negative in a formal way and hence appears as a negative slope. However, by advancing the optical delay such that the reference pulse travels a longer distance, we can prove directly that the pulses advance along the $\mathrm{PhCW}$, i.e., in the positive $x$ direction. The experimental data therefore indicates that these modes have a positive group velocity and a negative phase velocity. Note that for the determination of the eigenfield distribution as well as the sign of the wave vector, i.e., the direction of the phase velocity, local measurements of the optical amplitude and phase of the type presented here are required, because the Bloch mode itself only exists inside the periodic structure.

In conclusion, we have measured multiple Brillouin zones in a W3 photonic crystal waveguide. Lines with both positive and negative slope are observed in one Brillouin zone. We demonstrated that modes with a negative slope have a positive group velocity and a negative phase velocity. Furthermore, for each individual mode observed in the band structure, we retrieved the modal distribution. A comparison of wave vectors separated by the reciprocal lattice constant demonstrates that the modal distribution is different for individual Bloch harmonics. It is envisioned that this demonstration of probing both the eigenfield distribution and the band structure information of a photonic crystal waveguide and the determination of the relative sign of wave vector and the group velocity will open up further experimentation in areas such as optical Bloch oscillations [26], quasicrystals [27], negative refraction and left-handed behavior [28], and many others.

The authors thank H. G. Muller for useful discussions. This research is part of the strategic Research Orientation on Advanced Photonic Structures of the MESA ${ }^{+}$Institute for Nanotechnology. Furthermore, this work is part of the research program of the Stichting voor Fundamenteel Onderzoek der Materie (FOM), financially supported by the Nederlandse Organisatie voor Wetenschappelijk Onderzoek (NWO). W. B. is supported by the European Union as part of the IST-PICCO Project. T. J. K. acknowledges support by Agilent Technologies.

*Current address: Interdisciplinary Nanoscience Center (iNANO), Center for Atomic-scale Materials Physics
(CAMP), and Department of Physics and Astronomy, University of Aarhus, DK 8000 Aarhus C, Denmark.

Corresponding author.

Electronic address: L.Kuipers@amolf.nl

[1] J. D. Joannopoulos, R. D. Meade, and J. N. Winn, Photonic Crystals: Molding the Flow of Light (Princeton University Press, Princeton, NY, 1995).

[2] See, for example, Photonic Crystals and Light Localization in the 21st Century, NATO Science Series, edited by C. M. Soukoulis (Kluwer Academic, Dordrecht, The Netherlands, 2001).

[3] H. Benisty, J. Appl. Phys. 79, 7483 (1996).

[4] T.F. Krauss, R.M. De La Rue, and S. Brand, Nature (London) 383, 699 (1996).

[5] S. G. Johnson et al., Phys. Rev. B 62, 8212 (2000).

[6] S. Olivier et al., Phys. Rev. B 63, 113311 (2001).

[7] M. Agio and C. M. Soukoulis, Phys. Rev. E 64, 055603(R) (2001).

[8] S. J. McNab, N. Moll, and Y. A. Vlasov, Opt. Express 11, 2927 (2003).

[9] M. Lončar et al., Appl. Phys. Lett. 80, 1689 (2002).

[10] For a recent review, see V. Sandoghdar et al., in Photonic Crystals: Advances in Design, Fabrication, and Characterization (Wiley-VCH, Weinheim, Germany, 2004).

[11] S. Fan, I. Appelbaum, and J. D. Joannopoulos, Appl. Phys. Lett. 75, 3461 (1999).

[12] S. I. Bozhevolnyi et al., Phys. Rev. B 66, 235204 (2002).

[13] E. Flück et al., Phys. Rev. E 68, 015601(R) (2003).

[14] K. Okamoto et al., Appl. Phys. Lett. 82, 1676 (2003).

[15] P. Kramper et al., Phys. Rev. Lett. 92, 113903 (2004).

[16] M. L. M. Balistreri et al., Phys. Rev. Lett. 85, 294 (2000).

[17] M. L. M. Balistreri et al., J. Lightwave Technol. 19, 1169 (2001).

[18] A. Nesci et al., Opt. Commun. 205, 229 (2002).

[19] M. L. M. Balistreri et al., Science 294, 1080 (2001).

[20] H. Gersen et al., Phys. Rev. E 68, 026604 (2003).

[21] W. Bogaerts et al., Opt. Express 12, 1583 (2004).

[22] P. St. J. Russell, Appl. Phys. B 39, 231 (1986).

[23] Preliminary finite-difference time domain calculations show the same behavior.

[24] If we choose the phase of the reference branch as 0 , the LIA signals correspond to $\sin (k x)$ and $\cos (k x)$. Replacing $k$ by $-k$ directly reveals the $180^{\circ}$ phase shift.

[25] J. Witzens, M. Lončar, and A. Scherer, IEEE J. Sel. Top. Quantum Electron. 8, 1246 (2002).

[26] R. Sapienza et al., Phys. Rev. Lett. 91, 263902 (2003).

[27] L. Dal Negro et al., Phys. Rev. Lett. 90, 055501 (2003).

[28] S. Foteinopoulou and C. M. Soukoulis, Phys. Rev. B 67, 235107 (2003). 